\title{
Exploring patients' perceptions for insulin therapy in type 2 diabetes: a Brazilian and Canadian qualitative study
}

This article was published in the following Dove Press journal:

Patient Preference and Adherence

25 June 2010

Number of times this article has been viewed

\section{Camila Guimarães ${ }^{2}$ \\ Carlo A Marra' \\ Sabrina Gill' \\ Graydon Meneilly' \\ Scot Simpson ${ }^{3}$ \\ Ana LPC Godoy ${ }^{2}$ \\ Maria Cristina Foss de \\ Freitas $^{2}$ \\ Regina HC Queiroz ${ }^{2}$ \\ Larry Lynd'}

'The University of British Columbia, Canada; ${ }^{2}$ University of São Paulo, Brazil; ${ }^{3}$ The University of Alberta, Canada
Correspondence: Larry Lynd

University of British Columbia, Faculty of Pharmaceutical Sciences, 2I 46 East Mall,

Vancouver, BC,V6T IZ3, Canada

Tel + I 604-827-3397

Fax + | 604-827-40|4

Email llynd@interchange.ubc.ca
Objective: To explore which attributes of insulin therapy drive patients' preferences for management in Canada and Brazil.

Methods: A qualitative design was implemented in which a total of 32 patients with type 2 diabetes from Canada and Brazil, were interviewed in one of the 4 focus groups, or 16 individual interviews. Eighteen participants (56\%) were women and fourteen participants (44\%) were men (15 insulin nonusers and 17 insulin users). Two focus groups of 4 participants each and 9 individual interviews were conducted in Brazil. In Canada, 2 focus groups of 4 participants each and 7 individual interviews were conducted. A framework analysis was used to analyse all data.

Results: Brazilian participants, when considering two insulin treatments, would prefer the one that had fewer side-effects (specially hypoglycemia events), was noninjectable, had the lowest cost and was most effective. Meanwhile, Canadian participants would prefer a treatment that had fewer side-effects (specially weight gain), was less invasive, was more convenient and was most effective.

Conclusions: Finding the insulin-delivery system and the attributes of insulin therapy that best meet patients' preferences may lead to improved control, through improved compliance, which may ultimately reduce the financial burden of the disease and improve quality of life.

Keywords: type 2 diabetes, insulin administration, glycemic control, weight gain, hypoglycemia, qualitative study, patients' preferences

\section{Introduction}

Diabetes mellitus is one of the most common noncommunicable diseases globally, and exerts a substantial burden on patients, society and the health care system. Approximately 1 million (4.8\%) Canadians aged 20 years and older had diabetes in 1998/99, ${ }^{1}$ and this number is expected to increase to 2.4 million patients in $2016 .{ }^{2}$ Therefore, if the prevalence of diabetes follows current trends, health care costs for people with diabetes in Canada will increase by $75 \%$ between 2000 and $2016 .{ }^{2}$ Currently, 4.6 million people have diabetes in Brazil and if nothing is done to slow this epidemic, this number is expected to exceed 11.3 million by $2030 .^{3}$ Therefore, the resulting diabetes related morbidity and mortality will continue to pose an economic burden worldwide. ${ }^{3,4}$

Many of the complications and their associated costs attributable to diabetes are preventable. Increasing the effectiveness of surveillance and treatment for those who have the disease has been shown to be effective in decreasing long-term costs as a result of delayed or prevented complications. ${ }^{4}$ Improved blood-glucose control remains the goal of therapy for patients in order to prevent the development of diabetes complications that can substantially impact patients' longevity and quality of life, and increase health care costs. 
Despite advances in development of insulin analogs that has greatly improved the clinical effectiveness of insulin therapy, the goal of improved blood-glucose control remains elusive as many patients are either not using subcutaneous insulin therapy at all, or are noncompliant. ${ }^{5}$ Lack of diabetes education, inconvenience of repeated daily injections, fear of needles, injection-related anxiety, denial, and feeling that the disease has progressed are often major contributing factors. ${ }^{6-10}$ In this context, patients may be more likely to initiate insulin therapy if an alternative to the injectable route was available. ${ }^{9}$

Alternative routes of insulin delivery that are more clinically effective, more tolerable, and physiologically more like endogenous insulin are continously being investigated. The two most clinically promising options to date are oral and pulmonary insulin delivery system. Researchers have tried several approaches to overcome gastrointestinal metabolism and promote the bioavailability of oral insulin. The most promising oral insulin to date is hexyl-insulin-monoconjugate-2 (HIM-2), a recombinant insulin that has alterations in physiochemical characteristics that resist enzymatic degradation and facilitate absorption. Preclinical safety and pharmacokinetic data for HIM-2 are beginning to emerge and ongoing phase I and II clinical trials suggest that it may produce an acceptable glucose-lowering effect. In addition, because oral HIM-2 is delivered to the liver through the portal circulation thereby reproducing the physiological route of insulin secretion, oral HIM-2 may result in avoidance of pheripheral hyperinsulinemia. ${ }^{11}$ Pulmonary delivery of insulin is feasible given the large surface area and high permeability of the lungs where insulin can be effectively absorbed via the pulmonary alveoli. ${ }^{12}$ However, the inhalable insulin, Exubera ${ }^{\circledR}$, approved for use in 2006 as the first available alternative to insulin injections, was taken off the market less than two years later due to limited adoption by patients. Barriers may have been the cumbersome delivery system, safety concerns, and cost of the therapy, but this has not been explored. Attempts to overcome these problems have been made by other companies that are developing their own versions of inhaled insulin and promising results regarding patients' acceptance are beginning to emerge. ${ }^{13}$

The traditional approach to the physician-patient relationship in medical care, which focuses on disease rather than the patient, is usually ineffective in assisting patients to adequately manage their chronic illness. A partnership between the patient and physician is a model of patient-centred care that points at the importance of patients' participation in the decision-making process, and has been shown to increase adherence to management protocols, reduce morbidity, and improve quality of life of the patients with chronic disease. ${ }^{14}$ In addition, because diabetes is a self-managed disease, for diabetes care to succeed patients must be able to make decisions about how they will live with their illness. ${ }^{15}$ For health care providers to sucessfully facilitate patients' compliance which in turn will lead to increased effectiveness and less burden of disease, they will need to incorporate patients' preferences into diabetes treatment decision-making. ${ }^{16,17}$ Although the importance of the concordance model has been recognized in the literature ${ }^{18}$ and studies are emerging on patients' perceptions of diabetes therapy, ${ }^{16-19}$ formal valuation and quantification of patient's preferences for insulin therapy, and how they may be willing to trade off different attributes of insulin therapy, remains scarce.

The health care system, access to specific types of health care and drug therapy, and individual experiences with how they receive care for their chronic diseases is likely to influence patients' preferences for different aspects of therapy. Thus, we sought to elicit patients' preferences for insulin management of diabetes in two countries with very different health care systems, access to medications, and diabetesrelated outcomes. For example, in Brazil the public health care system does not cover all available drug therapies. For diabetic patients, it only covers some oral medication, NPH and regular insulins, glucose testing strips, and vials and syringes. Otherwise, other insulin devices (ie, pens, pump) as well as other insulins (ie, lantus, detemir) are available mainly for those who can afford to buy them, which means that most Brazilians use vials and syringes. Conversely, in Canada medical insurance coverage generally includes the costs of most therapies. In terms of insulin therapy, Canada and Brazil differ significantly in that insulin therapy in Canada primarily involves insulin pens compared to Brazil where insulin treatment still entails the use of needles, syringes, and multidose insulin vials. For these and other reasons it was therefore felt that patients with diabetes could potentially have different preferences for insulin therapy. One Canadian study has attempted to quantify patients willingness to pay for inhaled insulin using a contingent valuation methodology, ${ }^{20}$ while in Brazil no study has been identified. Therefore, the purpose of this study was to understand and compare which attributes of insulin therapy drive patients' preferences for management in both Canada and Brazil. The results of this study will then be used to quantify patients' preferences for insulin therapy, and to determine the relative importance that patients with type 2 diabetes place on different treatment attributes, using a discrete choice experiment (DCE).

\section{Methods}

A qualitative descriptive design was used in which focus group and individual interviews were conducted to elicit data 
from patients with type 2 diabetes. The practice of qualitative analysis is based upon two assumptions. The first is epistemological and states that qualitative researchers "believe that a phenomenon should be viewed in context", ${ }^{21}$ meaning that a researcher should be gathering knowledge/experiences from stakeholders in their own words. The second assumption is ontological and suggests that "there is not a single [all encompassing] reality apart from our perceptions", ${ }^{21}$ which suggests that to have an understanding of the impression of insulin use among type 2 diabetics, it is important to gather their words and ideas from them. With respect to this study specifically, the importance of these assumptions necessitated speaking directly with people with type 2 diabetes to begin to understand their views and perceptions of insulin therapy.

Focus group and individual interviews are becoming increasingly popular in health research for involving individuals in care management, treatment decision-making process, needs assessment, and health promotion. ${ }^{22,23}$ Focus group methodology is a form of group interview that capitalizes on interaction between research participants in order to explore the issues of importance to them based on each others' experiences and point of view, and pursuing their own priorities. ${ }^{23,24}$ In contrast, individual interviews are guided, one-to-one sessions. Therefore, both methods facilitate understanding participants' perceptions that may be helpful in identifying variables and hypotheses for quantitative research. However, these two methods are not substitutes but rather complementary. ${ }^{25}$ Because of that, both methods were used to provide complementary information leading to the identification of the most important attributes of insulin therapy, including the potential risks and benefits, that drive patients' treatment preferences, using their own vocabulary.

Ethics approval was obtained from both University of British Columbia-Providence Health Care Research Ethics Board and University of São Paulo Ethics Committee. Participants were asked to sign a written informed consent for study entry.

\section{Recruitment and data collection}

Focus group and individual interviews were conducted in both Canada and Brazil. A random sample was composed of type 2 diabetic men and women who attended a local diabetes education clinic at St Paul's Hospital in Vancouver, B.C, Canada, and a local hospital in Ribeirão Preto, São Paulo, Brazil. Patients were eligible for enrolment if they were 19 years of age or older, had physician-diagnosed type 2 diabetes, and were being actively treated with either an oral hypoglycemic, insulin, or both. The recruiting strategy included a personal approach involving an explanation of the study to potential participants by a member of the research team (CG) as well as using recruitment posters. In B.C, Canada, all patients are required to attend a diabetes education program before the costs of their glucose testing strips will be covered. In Ribeirão Preto, Brazil, all patients are required to be registered in the public heath care system before they are given their glucose testing strips without any costs. Therefore, participants in this study are expected to be representative of all type 2 diabetics.

An initial discussion guide was designed to help to create an open enviroment, and to facilitate the successful completion of the interviews by collecting as much information as possible regarding patients' perceptions of insulin therapy. The moderator used the same interviewer guide to conduct the focus groups and individual interviews in both countries. Initially, the moderator briefly gave baseline information regarding insulin therapy and potential alternatives for the subcutaneous route so all patients would have a similar baseline knowledge regarding the issues to be discussed. The moderator encouraged discussion of the widest possible range of attributes associated with insulin therapy with open ended questions supplemented with follow-up questions following some discussion. Both the focus groups and individual interviews were conducted in the same place patients were recruited, and typically lasted 30-50 minutes. All individual interviews and focus groups were audio recorded, transcribed and analyzed.

Given that many diabetes treatment options are assocaited with both potential risk and benefits, issues for discussion raised by the moderator included the pros (benefits) and cons (risk) of insulin therapy such as potential side effects of diabetes oral medication and insulin, emotional problems associated with insulin use or starting insulin treatment, cost of diabetes treatment, convenience of insulin administration, dislikes of diabetes medication, concerns about starting insulin treatment, alternative routes for insulin administration and the most important attributes of insulin therapy. All focus groups were composed of either insulin users or insulin nonusers because we hypothesized a priori that patients in each group would have different experiences and perceptions. Because of this difference, not all the issues were discussed in each group. Data collection continued until no new information resulted from the focus group and individual interviews, and it was deemed that all major themes were saturated. ${ }^{26}$

\section{Data analysis}

A framework analysis was used to analyse all data. ${ }^{27}$ Study data included notes from the moderator and participants, and the audio files and the transcripts from both individual and 
focus group interviews. The process of data analysis began during the data collection, by facilitating the discussion in order to generate the richest data possible. This provided a complete record of the interviews and facilitated analysis of the data. ${ }^{28}$ The familiarization with the data was achieved by listening to the audio recorded files and reading the transcripts as well as the moderator and particpants' notes several times to get a sense of each interview as a whole before breaking it into parts. Ideas, notes and short sentences that arose from the transcripts were written in the margin of the pages and the major themes begun to emerge. Relevant quotes were also highlighted in the transcripts.

All relevant quotes, ideas and notes that arose from the data were then re-arranged under the newly developed main themes. This was to achieve data reduction by putting relevant information together and deleting data that did not relate to insulin therapy. Each participant was asked to answer a final summary question in which he/she should list the most important atrributes of the insulin treatment for them, in order of importance, taking into account everything that was discussed in the interview. These follow up probes were also analysed by the researchers to consider the consistency and the specificity of responses. In addition, for interpreting the data, words, context, internal consistency, the frequency and extensiveness of comments were also considered. ${ }^{24}$

\section{Results}

A total of 32 patients with type 2 diabetes from both countries were interviewed in one of the 4 focus groups or 16 individual interviews. Eighteen participants (56\%) were women, and 15 (47\%) were insulin naïve. Two focus groups of 4 participants each and 9 individual interviews were conducted in Brazil. In Canada, 2 focus groups of 4 participants each and 7 individual interviews were conducted. The mean age of the sample was 58 , ranging from 32 to 85 years. In both countries, similar themes emerged from the interviews. Major themes identified that drive patients' preferences for management included sideeffects, effectiveness and cost of insulin therapy. Participants also seek treatment approaches that allow the elimination of injections, particularly in Brazil. In Canada, convenience was another important theme for patients. Table 1 shows a summary of the main results.

\section{Side effects}

The theme of side effects reflected participants' desire to experience fewer hypoglycemic events and less weight gain. However, hypoglycemia appeared to be more disturbing for Brazilians, while weight gain emerged more frequently among Canadians. In Brazil, the importance of hypoglycemia events can be illustrated by the comments of two participants: "I take insulin three times a day an I still can not have my blood sugar controlled, I have many events of hypoglycemia and it is very disturbing”. 59-year old female, insulin user. Another stated, "I have been much more controlled after I started with insulin, and my biggest complaint today is the hypoglycemia events I have during the night'. 31-year-old male, insulin user. Hypoglycemic events were also important for the insulin naïve as can be illustrated by the comment of one participant: "I am feeling good, without any hypoglycemia or hyperglycemia events, there is no need for me to use insulin". 61-year-old female.

In Canada, the comments of many participants illustrate patient's desire to avoid weight gain: "I would have to agree that the weight gain is one of the factors I didn't like about insulin. That normal weight gain is an issue”. 53-year-old male, insulin user. Another participant stated: "Weight gain is of course the worst side effect. And that's been an emotional problem". 50-year-old male, insulin user. Another participant said: "I do experience weight gain, from the insulin itself. That is uncomfortable, I don't like that feeling. I'd rather be on a pill, rather than the insulin, for that weight gain factor". 46-year-old female, insulin user. In addition to the weight gain, hypoglycemia emerged as another disturbing side effect: "If I have one immediate fear of diabetes, it's hypoglycemia”. 69-year-old-female, insulin-naïve. Another said: "I do experience hypoglycemia, maybe once or twice a month. Um, that's really uncomfortable”. 46-year-old female, insulin user.

\section{Avoidance of injections}

A strong desire to avoid injections was the most frequent issue raised by Brazilian participants when asked what they believe would be important for them when it comes to insulin therapy. Many insulin naïve spoke about their fear of injections: "There is no way I will start insulin, I can not inject myself, I feel terrified just to think about the injections". 62-year-old male. Another stated, "I do not feel just fear of the injections, I feel terrified of them". 58-year-old female. Interestingly, some insulin users shared the same opinion as illustrated by this comment, "I take insulin twice a day but I just can not inject myself, I have fear of the injections and my friend's husband is the one who injects for me". 64-year-old female. Another stated, "I am better controlled with insulin, but I simply hate the injections, I wish I could get rid of them ". 58-year-old male. For those taking insulin, when asked about their dislikes of insulin therapy, they also spoke about the pain and body injuries associated with the 
Table I Major themes identified that drive patients' preferences for insulin therapy

\section{Side-effects}

"I have been much more controlled after I started with insulin, and my biggest complaint today is the hypoglycaemia events I have during the night".

3I-year-old Brazilian male

"I do experience weight gain, from the insulin itself. That is uncomfortable, I don't like that feeling. l'd rather be on a pill, rather than the insulin, for that weight gain factor."

46-year-old Canadian female

\section{Avoid injections}

"I am better controlled with insulin, but I simply hate the injections, I wish I could get rid of them."

58-year-old Brazilian male

"I would do the impossible to avoid the injections, they are very inconvenient and because I am not very careful with changing the local of the injections, I am usually full of hard spots."

58-year-old Brazilian male

"If a pill was available it would improve a lot my life, I hate the injections, they are like a bad kharma in my life."

33-year-old Brazilian female

"If I lose control, where l'm forced to take insulin, then I'm gonna look at the alternatives of how l'm gonna take it. What l'm saying is I don't wanna shoot myself if there's something that I could just breathe it in."

48-year-old Canadian male

\section{Effectiveness}

"I have been much better controlled after I started insulin I feel less thirsty and in a better mood, but there's no way I like the injections."

64-year-old Brazilian female

"I guess the most important thing for me is the control of my blood sugars and what goes with that. That you're feeling better, that l'm not running to the bathroom to pee. And, it improves your quality of life."

50-year-old Canadian male

"I take insulin because I fear the complications of my diabetes, and I know the insulin can help me to avoid them."

42-year-old Brazilian female

\section{Cost}

"In order to be a healthier person, if it means taking insulin l'll take the insulin, but I'm gonna search high and low, for the most practical and least invasive means of accepting it. I don't care about the cost. It could cost me half of my salary."

48-year-old Canadian male "If I have to pay extra I would continue with the injections because I can't afford extra expense, but if a pill was covered from the government the same way my injections are, of course I would prefer the pill."

58-year-old Brazilian male

"If a pill was available I would do what I could to buy it because I take insulin three times a day and it is very uncomfortable, it hurts."

42-year-old Brazilian female

\section{Convenience}

"Well, I take my insulin 5 times a day. So, it is inconvenient if I'm out somewhere shopping, and stuff like that. And sometimes I just get tired of doing it. It's a lot of extra work."

55-year-old Canadian female

"What I don't like is having to stick to that strict regiment of taking it early in the morning, and having breakfast, and then having to take it at 5 o'clock, and having supper. More convenience, not having such a regimented schedule would be nice."

46-year-old Canadian female "I'm doing my best to take my insulin on time, every day. But sometimes I do forget, and sometimes I'm not home, at the right time to do my insulin. It cause some inconvenience sometimes, specially when I travel. You know, I can't be on time for my insulin, and it's a trouble, even the transportation sometimes."

72-year-old Canadian male

injections: "I take insulin three times a day and I feel the pain very inconvenient". 42-year-old female. Another said, "I would do the impossible to avoid the injections, they are very inconvenient and because I am not very careful with changing the local of the injections, I am usually full of hard spots". 58-year-old male. There was a strong and consistent preference for an oral insulin formulation to make the treatment more convenient and particularly to eliminate the injections: "A pill would be much better. It would facilitate a lot my life". 31-year-old male. Another said, "If a pill was available it would improve a lot my life. I hate the injections, they are like a bad karma in my life". 33-year-old female. 
In Canada, concerns and dislikes related to insulin therapy included fear of the needles as well as the pain and body injuries associated with the injections: "The part of my insulin treatment I don't like, of course, is stickin' myself with needles. Sometimes it hurts when you give yourself an injection. I would like to get off it, but we are heading that direction". 59-year-old man, insulin user. Another participant said: "I don't want to take insulin mainly because I am a little bit scared about needles". 70-year-old female. Another stated: "The only thing that I dislike is the bruising. That you would see the marks. And that people go, 'What's that on you? '”. 50-year-old male. Many patients also showed a strong desire for another route of administration to avoid injections: "If I lose control, where I'm forced to take insulin, then I'm gonna look at the alternatives of how I'm gonna take it. What I'm saying is I don't wanna shoot myself if there's something that I could just breathe it in". 48-year-old male. Another participant said: "If they can find something that could eliminate the injections. It will be great if they can find an oral insulin, that you just swallow, and, you know, that would be great". 63-year-old man, insulin user. Another participant said: "Inever liked the needles, it cause me a little bit of pain. I really don't like it. Well, I hope that something can replace insulin in the future, and so, that I don't have to have injections. Something like oral would be even better, because at least I can't feel that needle pain". 72-year-old male, insulin user.

\section{Effectiveness}

In both countries, the theme of effectiveness reflected patients' improvement in well being with insulin therapy as well as patients' awareness of the benefits of insulin treatment. In addition, some Brazilians also stated that they take insulin because they fear the complication of their diabetes.

In Brazil, many of the insulin users stated that they do not like the injections but they stand it because they feel better when they take insulin: "I have been much better controlled after I started insulin, that's why I take it, I feel less thirsty and in a better mood, but there's no way I like the injections". 64-year-old female. Another stated, "I take insuln just because it helps me to decrease my blood sugar and then I feel better". 58-year-old male. Some participants stated that they take insulin because they fear the complication of their diabetes: "I take insulin because I had heart problems and I fear the complications of my diabetes, and I know insulin can help me to avoid them". 42-year-old female. Another said, "The main reason I take insulin is because it's what keeps me alive, and if I don't take it, my diabetes will complicate and I don 't want that'. 31-year-old male.
In Canada, the importance of effectiveness can also be illustrated by the following comment: "The most important thing is getting the blood sugar down to the ideal level". 53-year-old Canadian male. Some patients, similarly to the Brazilian patients, associated the blood glucose control with an improvement in well being, as one participant stated: "I guess the most important thing for me is the control of my blood sugars and what goes with that. That I'm feeling better, that I'm not running to the bathroom to pee, and all these things. I'm in control, I'm feeling better, there's nothing better than that. And, it improves your quality of life, so getting better control makes a big difference”. 50-year-old male, insulin user.

\section{Cost}

Many participants had no out-of-pocket expenses related to their diabetes medication treatment at the time of the study. However, despite this, cost emerged as one of the most important attributes related to the insulin therapy in both countries, although in different ways. In Brazil, although patients' desire to avoid injections, have less side-effects, and for an alternative and more convenient route of insulin administration, many participants were not willing to pay out-of-pocket for them. The comments of two participants clearly reveal the problem: "If I have to pay extra I would continue with the injections because I can't afford extra expenses, but if a pill was available with no costs the same way my injections are, of course I would prefer the pill”. LCP, 58-year-old male. The participants that were willing to pay out-of-pocket stated that they would pay for an oral route: "If a pill was available I would do what I could to buy it because I take insulin three times a day and it is very unconfortable, it hurts". MLNP, 42-year-old female.

When the Canadian patients were asked if they would be willing to pay extra for any of the insulin therapy attributes, they said that they were willing to pay out-of-pocket for an alternative route to eliminate injections, for improved convenience, to feel better. One participant said: "If my condition was to deteriorate, I would be willing to pay more to feel good”. MAP, 69-year-old female, insulin naïve. Another stated: "I would be willing to pay more for improved convenience”. GHT, 50-year-old male, insulin user. Another participant said: "I think paying more would justify having more convenience, definitely". SEMB, 46-year-old female, insulin user. Among the insulin naïve participants, some said that they would not have a problem taking insulin, but they would prefer the more convenient way as possible: "In order to be a healthier person, if it means taking insulin I'll take insulin, but I'm gonna search high and low, for the most 
practical and least invasive means of accepting it. I don't care about the cost. It could cost me half of my salary". RAY, 48-year-old male.

\section{Convenience}

In Canada, many insulin users raised the inconvenience of using insulin when asked about their dislikes related to the insulin therapy. Many patients spoke about the inconvenience in public places, the strict schedule, the inconvenience when travelling, and the frequency of administration: "Well, I take my insulin 5 times a day. So, it is inconvenient if I'm out somewhere shopping, and stuff like that. And sometimes I just get tired of doing it. It's a lot of extra work". MAG, 55-year-old female. Another stated: "For me, what I don't like about insulin is the inconvenience. Having to stick to that strict regiment of taking it early in the morning, and having breakfast, and then having to take it at 5 o'clock, and having supper. I don't like that strict regiment. That's about the biggest thing for me. More convenience, not having such a regimented schedule would be nice". SEMB, 46-year-old female. Another participant said: "I'm doing my best to take my insulin on time, every day. But sometimes I do forget, and sometimes I'm not home, at the right time to do my insulin. It causes some inconvenience sometimes, specially when I travel. You know, I can not be on time for my insulin, and it's a trouble, even the transportation sometimes". JK, 72-year-old male. Another stated: "Like when I go to a party, or gathering, I have to go to a certain place, or even in front of the public, open up my shirt, and do the insulin, and, I didn't like to do it, those kind of things like the insulin with the public, but sometimes I have no choice, and there is no place to go to do it". JM, 63-year-old male.

\section{Discussion}

Individual and focus group interviews proved to be efficient techniques of exploring patients' perceptions of insulin therapy among type 2 diabetics. Overall, participants from both countries identified similar attributes as major contributors to their preferences in regards to insulin therapy. In general, when considering insulin treatments, patients in this study would prefer a treatment with the fewest side-effects (in particular, weight gain and hypoglycemic events), does not require injection, and is the least costly and the most effective. In Canada, convenience was another major theme that emerged from the interviews. Brazilian participants were more emphatic on avoidance of injections compared to Canadians. These findings are in accordance with previous studies that have shown that treatment preferences for insulin therapy seem to be largely based on the side-effects, effectiveness, convenience and cost. ${ }^{20,29-31}$

Some basic background information regarding insulin therapy and alternative routes of insulin administration was provided to all participants at the beginning of all interviews to ensure that all participants had the same baseline knowledge regarding the issues of interest. Interestingly, the majority of insulin users from both countries were not aware of the potential alternative routes of insulin administration and most insulin naïve patients had very little general knowledge of insulin therapy in general. Some participants did not even understand that diabetes is a progressive disease and that insulin use is likely in their future: "I am well controlled and hopefully I will never need the injections, I fear them". NLC, 72-year-old Brazilian female. Another said: "If you're gone to number 2 diabetes, what are the chances that you will have to take insulin, eventually?" MOB, 85-year-old Canadian female.

In addition to the main themes that emerged from the interviews, Canadian participants also reported some kind of emotional problem related to their treatment such as feelings of failure, guilt, feelings that the disease had progressed, and fear of the complications of the disease. These findings support the idea that diabetes treatment is a psychological burden for many patients. ${ }^{6,32,33}$ In addition, many patients from both countries also believed insulin to be the last resort to manage diabetes, and they associated insulin with the progression of the desease: "But with type 2 diabetes I feel I'm early in the disease, and there's not that sense of urgency and fear. Whereas, if I start to take insulin, then I realize, I'm getting closer to the end. I'm just hoping that I don't get that far." RP, 47-year-old Canadian male. This finding is in accordance with previous studies, ${ }^{6,8}$ and may help to understand why many patients either delay or refuse to start insulin therapy if at all possible.

Some Canadians, particularly the elderly participants, reported concerns related to aging when considering initiating insulin use: "Withdrawing insulin from the bottle, even getting the needle into the bottle. So that would be a concern for me. The older I get, the less mentally acute I become". MAP, 69-year-old female. Another stated: "I hope I don't go into insulin, because I notice as I grow older, I'm 85, that my hands get shaky, and I've also lost the strength in my hands". MOB, 85-year-old female. This finding suggests that an alternative route of administration may benefit many patients that feel they are unable to self-inject.

Participants from both countries showed a strong desire to avoid the injections, however, Brazilians appeared to me more emphatic, when compared to Canadians. This may be explained by the fact that most of the Brazilian participants were using 
vials and syringes at the time of the study, while most of the Canadians were using insulin pens, which have been shown to be less painful and more convenient:" "When I first had to use the syringes, I didn't like that at all. I felt ashamed to use that. I felt like a drug addict or something. So I find the pen much more acceptable". MAG, 55-year-old female.

Another issue frequently raised by participants from both countries was a strong desire for an oral route, specially to make the treatment more convenient and to eliminate injections. Many Canadians said that they were willing to pay outof-pocket for an alternative route, for more convenience, and to eliminate injections. On the other hand, although Brazilians' preferred to avoid injections experience fewer side-effects, most were not willing to pay out-of-pocket for them. The few Brazilian participants that were willing to pay out-of-pocket stated that they would pay for an oral route for insulin administration. Despite this strong desirability to avoid injections, it is interesting to note that many patients agreed that insulin therapy is the best treatment to achieve blood glucose control. This finding has been reported elsewhere. ${ }^{19}$

In general, patients from both countries showed an overall dislike for the insulin therapy and the most raised reasons were: the rigid schedule and inconvient time and frequency of administration, the pain and body injuries associated with the injections, the inconvenience in public places, the fear of injections, feelings that the disease had progressed, and the weight gain and higher frequency of hypoglycemia. Brazilian patients also reported that health providers paid little attention to their concerns regarding diabetes treatment. However, if measures are to be taken to improve compliance, these should be based on a deeper understanding of patient's experiences of their treatment rather than the perceptions of health care providers. ${ }^{18}$

A large ongoing North American trial of diabetes, called ACCORD, designed to intensively lower blood glucose of adults with type 2 diabetes at high risk of heart disease has been halted because of an increased number of deaths among those receiving intensive treatment compared to those receiving less-intensive standard treatment. This finding was unexpected as it is well established that an HbA1c goal of less than seven percent reduces microvascular complications resulting from diabetes. Researchers have not determined a specific cause for the increased deaths among the intensive treatment group, but the results from ACCORD study strengthen the importance the individualization of treatment to determine what the treatment goals should be for a specific patient with specific characteristics. ${ }^{35}$ Therefore, for health care providers to facilitate improved diabetes management and treatment adherence, which in turn is expected to improve treatment effectiveness, reduce long term complications and improve patient outcomes, they will need to incorporate patients' preferences into their decisions in regards to diabetes management. ${ }^{16,17}$

Now that we have identified the most important attributes of the insulin therapy from patients' perspectives in both countries, we will move forward to quantify the value patients place on each attribute, in monetary terms, and the rate at which they would be willing to accept trade-offs among insulin therapy attributes. This ongoing study will be, to the best of our knowledge, the first discrete choice experiment (DCE) to simultaneously quantify the cost-utility of different attributes of insulin therapy such as effectiveness, side-effects, convenience and route of administration.

\section{Conclusions}

Although the relationship between medication adherence and health outcomes is complex, our findings suggest that open and ongoing discussions that addresses patients' concerns regarding potential side-effects, effectiveness, convenience, route of administration, and potential cost of insulin therapy need to be considered, particularly when trying to initiate insulin therapy. In particular, we found that an alternative to the injectable insulin may mitigate some of the resistance to initiating insulin therapy and improve treatment adherence for many patients These findings, in conjunction with the removal of Exubera ${ }^{\circledR}$ from the market, further emphasize the need for continued investigation of alternative insulin-delivery systems that will better meet the needs and preferences of patients. In addition, we also determined patients' preferences for insulin therapy to be largely based on effectiveness, convenience, route of administration, side-effects and cost. Therefore, finding the insulin-delivery system and the attributes of insulin therapy that are constitent with patients' preferences may lead to improved control, through improved treatment adherence, which may ultimately reduce the financial burden of the disease and improve quality of life.

\section{Acknowledgments}

This study was supported by a grant from the University of British Columbia, Faculty of Pharmaceutical Sciences, and by CAPES (Coordenação de Aperfeiçoamento de Pessoal de Nível Superior). Dr Lynd is a Michael Smith Foundation for Health Research Scholar and a Canadian Institutes for Health Research New Investigator. Dr Marra is a Canada Research Chair in Pharmaceutical Outcomes Research. In addition, we would like to thank the staff from all diabetes education 
clinics in both Canada and Brazil for their co-operation during the data collection period.

\section{Disclosure}

The authors report no conflicts of interest in this work.

\section{References}

1. Public Health Agency of Canada. Diabetes in Canada, 2nd edn., Ottawa, Canada: Public Health Agency of Canada; 2002.

2. Ohinmaa A, Jacobs P, Simpson S, Johnson JA. The projection of prevalence and cost of diabetes in Canada: 2000 to 2016. Canadian Journal of Diabetes. 2004;28(2):116-123.

3. Wild S, Roglic G, Green A, Sicree R, King H. Global prevalence of diabetes: estimates for the year 2000 and projections for 2030. Diabetes Care. 2004;27:1047-1053.

4. Idf.org. [homepage on the internet]. International Diabetes Federation. Diabetes Atlas: Executive Summary. 2003, Second edn. Available from http://www.idf.org/e-atlas. Accessed September 15, 2006.

5. UKPDS. Intensive blood-glucose control with sulphonylureas or insulin compared with conventional treatment and risk of complications in patients with type 2 diabetes (UKPDS 33). UK Prospective Diabetes Study (UKPDS) Group. Lancet. 1998;352:837-853.

6. Korytkowski M. When oral agents fail: practical barriers to starting insulin. Int J Obes Relat Metab Disord. 2002;26(3):S18-24.

7. Zambanini A, Newson RB, Maisey M, Feher MD. Injection related anxiety in insulin-treated diabetes. Diabetes Res Clin Pract. 1999;46:239-246.

8. Peyrot M, Rubin RR, Lauritzen T, et al. Resistance to insulin therapy among patients and providers: results of the cross-national Diabetes Attitudes, Wishes, and Needs (DAWN) study. Diabetes Care. 2005;28: 2673-2679.

9. Cappelleri JC, Gerber RA, Kourides IA, Gelfand RA. Development and factor analysis of a questionnaire to measure patient satisfaction with injected and inhaled insulin for type 1 diabetes. Diabetes Care. 2000; 23:1799-1803.

10. Polonsky WH, Fisher L, Guzman S, Villa-Caballero L, Edelman SV. Psychological insulin resistance in patients with type 2 diabetes: the scope of the problem. Diabetes Care. 2005;28:2543-2545.

11. Clement S, Still JG, Kosutic G, McAllister RG. Oral insulin product hexyl-insulin monoconjugate 2 (HIM2) in type 1 diabetes mellitus: the glucose stabilization effects of HIM2. Diabetes Technol Ther. 2002;4: 459-466.

12. Cefalu WT, Skyler JS, Kourides IA, et al. Inhaled human insulin treatment in patients with type 2 diabetes mellitus. Ann Intern Med. 2001; 134:203-207.

13. Hayes RP, Muchmore D, Schmitke J. Effect of inhaled insulin on patient-reported outcomes and treatment preference in patients with type 1 diabetes. Curr Med Res Opin. 2007;23:435-442.

14. Bauman AE, Fardy HJ, Harris PG. (2003) Getting it right: why bother with patient-centred care? Med J Aust. 2003;179:253-256.

15. Funnell MM, Anderson RM. MSJAMA: the problem with compliance in diabetes. JAMA. 2000;284:1709.

16. Hayes RP, Bowman L, Monahan PO, et al. Understanding diabetes medications from the perspective of patients with type 2 diabetes: prerequisite to medication concordance. Diabetes Educ. 2006;32:404-414.

Patient Preference and Adherence

\section{Publish your work in this journal}

Patient Preference and Adherence is an international, peer-reviewed, open access journal that focusing on the growing importance of patient preference and adherence throughout the therapeutic continuum. Patient satisfaction, acceptability, quality of life, compliance, persistence and their role in developing new therapeutic modalities and compounds to
17. Kruger DF. Tying it all together: matching insulin regimens to individual patient needs. Diabetes Educ. 2007;33(4):91S-95S.

18. Vermeire E, Hearnshaw H, Van Royen P, Denekens J. Patient adherence to treatment: three decades of research. A comprehensive review. J Clin Pharm Ther. 2001;26:331-342.

19. Cefalu WT, Chantal M, Davidson J, Freemantle N, Gough S, Canovatchel W. Patients' perceptions of subcutaneous insulin in the OPTIMIZE Study: A multicenter follow-Up study. Diabetes Technol. Ther. 2008; 10:25-38

20. Sadri H, MacKeigan LD, Leiter LA, Einarson TR. Willingness to pay for inhaled insulin: a contingent valuation approach. Pharmacoeconomics. 2005;23:1215-1227.

21. Trochim WMK. The Research Methods Knowledge Base, 2nd edn. Cincinnati, Ohio: Atomic Dog Publishers; 2001.

22. Duke SS, Gordon-Sosby K, Reynolds KD, Gram IT. A study of breast cancer detection practices and beliefs in black women attending public health clinics. Health Educ Res. 1994;9:331-342.

23. Kitzinger J. Qualitative research. Introducing focus groups. BMJ. 1995; 311:299-302.

24. Rabiee F. Focus-group interview and data analysis. Proc Nutr Soc. 2004;63:655-660.

25. Kaplowitz MD, Hoehn JP. Do focus groups and individual interviews reveal the same information for natural resource valuation? Ecological Economics. 2001;36:237-247.

26. Giacomini MK, Cook DJ. Users' guides to the medical literature: XXIII. Qualitative research in health care B. What are the results and how do they help me care for my patients? Evidence-Based Medicine Working Group. JAMA. 2000;284:478-482.

27. Krueger R. Focus Groups: A Practical Guide for Applied Research. Thousand Oaks, CA: Sage Publications; 1994.

28. Lewis M. Focus group interviews in qualitative research: A review of the literature. Action Research E-Reports: 2000.

29. Summers KH, Szeinbach SL, Lenox SM. Preference for insulin delivery systems among current insulin users and nonusers. Clin Ther. 2004;26:1498-1505.

30. Freemantle N, Blonde L, Duhot D, et al. Availability of inhaled insulin promotes greater perceived acceptance of insulin therapy in patients with type 2 diabetes. Diabetes Care. 2005;28:427-428.

31. Hunt LM, Valenzuela MA, Pugh JA. NIDDM patients' fears and hopes about insulin therapy. The basis of patient reluctance. Diabetes Care. 1997;20:292-298.

32. Meece J. Dispelling myths and removing barriers about insulin in type 2 diabetes. Diabetes Educ. 2006;32:9S-18S.

33. Penckofer S, Ferrans CE, Velsor-Friedrich B, Savoy S. The psychological impact of living with diabetes: women's day-to-day experiences. Diabetes Educ. 2007;33:680-690.

34. Korytkowski M, Bell D, Jacobsen C, Suwannasari R. A multicenter, randomized, open-label, comparative, two-period crossover trial of preference, efficacy, and safety profiles of a prefilled, disposable pen and conventional vial/syringe for insulin injection in patients with type 1 or 2 diabetes mellitus. Clin Ther. 2003;25:2836-2848.

35. Nih.gov [homepage on the internet]. National Institutes of Health. For Safety, NHLBI Changes Intensive Boold Sugar Treatment Strategy in Clinical Trial of Diabetes and Cardiovascular Disease. U.S Department of Health and Human Services - National institutes of Health (NIH) Available from: www.nih.gov/news/health/feb2008/nhlbi-06.htm). Accessed February 26, 2008.

optimize clinical outcomes for existing disease states are major areas of interest. This journal has been accepted for indexing on PubMed Central. The manuscript management system is completely online and includes a very quick and fair peer-review system. Visit http://www.dovepress.com/ testimonials.php to read real quotes from published authors. 